\title{
Successful fast track protocol implementation for patients undergoing transapical transcatheter aortic valve implantation
}

Nestoras Papadopoulos ${ }^{1 * \dagger}$, Ali El-Sayed Ahmad ${ }^{1 \dagger}$, Marlene Thudt', Stephan Fichtlscherer ${ }^{2}$, Patrick Meybohm ${ }^{3}$, Christian Reyher ${ }^{3}$, Anton Moritz ${ }^{1}$ and Andreas Zierer ${ }^{1}$

\begin{abstract}
Background: The aim of the current study is to report our experience with fast-track treatment of patients undergoing transapical transcatheter aortic valve implantation (TA-TAVI) and to determine perioperative predictors for fast-track protocol failure.

Methods: Being one of the pioneering centers to start performing TA-TAVI back in 2005, we routinely included patients undergoing this procedure into our fast-track management program since 2008. Between January 2008 and June 2013, 207 consecutive high-risk patients (mean age $79 \pm 7$ years, mean Log. EuroSCORE 24 \pm 10 ) who underwent TA-TAVI accordingly to our institutional fast-track approach were prospectively collected and analyzed. Uni- and multivariate analysis were performed to identify independent pre- and perioperative predictors of fast-track protocol failure, defined as inability to discharge the patient from the intensive care unit (ICU) on the day of surgery or as readmission to the ICU $48 \mathrm{~h}$ after the initial discharge.

Results: Fast-track management was successful in $83 \%$ of the patients. 30-day mortality was $8 \%$. Fast-track protocol failure $(17 \%)$ was associated with an outcome worsening compared to the remaining patients (mortality: $40 \%$ vs. $2 \%$ and mean hospital stay: $19 \pm 12$ vs. $10 \pm 9$ days; $P=.002$ ). Independent predictors of fast-track protocol failure were age $\geq 85$ years (OR 3.1; Cl $95 \%$ 1.89-6.21), ejection fraction (EF) $\leq 30 \%$ (OR 2.6; Cl $95 \%$ 1.99-7.52), moderate to severe preoperative mitral valve regurgitation (OR 2.7; Cl $95 \% 1.27-6.43$ ) and fluoroscopy time $\geq 12$ min (OR 2.9; Cl 95 \% 1.28-7.46).

Conclusions: Fast-track patient management following TA-TAVI is safe and reproducible in the majority of patients. Besides patient-related preoperative risk factors (age $\geq 85$ years, EF $\leq 30 \%$ and moderate to severe preoperative mitral valve regurgitation) a technically challenging intraoperative course as evidenced in a prolonged fluoroscopy time are independent predictors of fast-track protocol failure which is associated with high loss of patient outcome.
\end{abstract}

Keywords: Heart valve replacement, Transapical, Percutaneous, Heart valve prosthesis (bioprosthetic), Cardiac catheterization/intervention

\footnotetext{
* Correspondence: nestoras.papadopoulos@gmail.com

${ }^{\dagger}$ Equal contributors

'Division of Thoracic and Cardiovascular Surgery, Johann-Wolfgang-Goethe University Frankfurt/Main, Theodor Stern Kai 7, 60590 Frankfurt am Main,

Germany

Full list of author information is available at the end of the article
} 


\section{Background}

Early pioneer work in 1980s launched the early extubation as a safe technique in a small series of patients undergoing cardiac surgery $[1,2]$. Since then, the continuously expanding impact of health economics on the patient management and the frequently limited financial resources have led to an increasing interest in fast-track treatment protocols following cardiac surgery [3].

Transapical transcatheter-based aortic valve implantation (TA-TAVI) has been identified as a safe and efficient alternative to classic surgery, especially in patients carrying an unacceptably high perioperative risk $[4,5]$. Due to the minimally invasive nature of this approach that eliminates sternotomy and the need of cardiopulmonary bypass (CPB), TA-TAVI patients may represent a very attractive cohort for fast-track treatment concepts.

Despite the continuous improvement of the surgical and anesthesiological management of patients in cardiac surgery and the insatiable thirst of medical departments for optimized use of intensive care capacities, TA-TAVI patients remain a cohort with high perioperative risk profile and limited biological reserves, who rarely "excuse mistakes" [6, 7]. Thus, the aim of the current study is to report our institutional experience following fast-track treatment of patients undergoing TA-TAVI and to identify predictors for potential fast-track protocol failure.

\section{Methods}

\section{Patient population}

TA-TAVI was introduced as a novel technique in our department in 2005 [8]. Fast-track protocol has been routinely applied for TA-TAVI patients since 2008. Between January 2008 and June 2013, 207 consecutive high-risk patients underwent TA-TAVI followed by the fast-track postoperative treatment approach. The choice of treatment was made at the discretion of the heart team, consisting of 2 cardiac surgeons, 2 interventional cardiologists and lately 2 anaesthesiologists. From 2007 on we assessed all TAVI candidates according to the established recommendations and guidelines in order to find the best possible TAVI approach for each individual patients. Patient data were collected prospectively during treatment using standardized forms to record demographic and clinical characteristics as well as procedural and follow-up data. The local Ethics Committee at the Hospital of the Johann Wolfgang Goethe University, Frankfurt/Main, Germany approved the study protocol and individual patient consent was waived.

\section{Preoperative anaesthesiologic management}

After insertion of a radial artery catheter for invasive monitoring of arterial pressure, anesthesia was introduced using sufentatil (Janssen, Neuss, Germany), disoprivan (Fresenius Kabi, Bad Homburg, Germany) as well as rocuronium (Essex pharma, Munich, Germany), and was maintained with disoprivan (Fresenius Kabi, Bad Homburg, Germany) and remifentanil (Braun, Melsungen, Germany). Oropharyngeal intubation and insertion of a central venous catheter (in the internal jugular vein) was performed afterwards. Mechanical ventilation with biphasic positive airway pressure was established after the intubation adjusted to the gas exchange (Dräger Oxylog 3000, Dräger Medical Germany GmbH, Lübeck, Germany). Further anesthesiologic management included a peripheral venous and urinary catheter. Despite continuous peripheral oxygen saturation control, respiration was monitored at fixed time intervals by blood gas analysis measurements.

\section{TA-TAVI procedure}

Our institutional protocol for TA-TAVI has been previously described in detail $[9,10]$. Briefly, all operations were performed in a specially equipped angiography suite that fulfils the standards of a hybrid operating room. Besides standard hemodynamic monitoring, transesophageal echocardiography and $\mathrm{CPB}$ were routinely available. A limited left anterolateral incision $(5-7 \mathrm{~cm})$, in the fifth intercostal space, was used to access the apex of the heart. A bipolar epicardial pacing wire was placed and tested. Two U stitches with Teflon felt pledgets using 3-0 Prolene polypropylene (Ethicon, Inc., Somerville, NJ) were placed in the apex of the left ventricle. They served as purse string for linear closure of the left ventricle at the end of the procedure. Following balloon valvuloplasty, all valve deployments were performed with standard volumetric inflation of the balloon. Fluoroscopy and transesophageal echocardiography were used to guide the catheter across the native valve and direct deployment of the stent at the level of the annulus. During deployment, the heart was unloaded with rapid ventricular pacing. Valve function was immediately assessed by angiographic and echocardiographic visualization. Intercostal blockade was performed with ropivacaine (Ratiopharm $\mathrm{GmbH}$, Ulm, Germany). The pericardium was partially closed over the apex and a left lateral chest tube inserted. The incision was closed in a standard fashion.

\section{ICU fast-track protocol}

All patients were transferred postoperatively intubated to the intensive care unit (ICU) where ECG, chest radiography and clinical laboratory test as well as blood gas analysis were immediately performed. The ICU is a 36bed unit run by full-time intensivists and cardiac surgeons with a 2:1 staff to patient ratio. Standard postoperative care consisted of mechanical ventilation, cardioacitve drugs if indicated, the use of warm air heaters to maintain noromothermia and analgesia with a combination of nonsteroidal anti-inflammatory drugs (paracetamol, Ratiopharm GmbH, Ulm, Germany) and intravenous 
morphine boluses (piritramid, Janssen-Cilag GmbH, Neuss, Germany) as required. Blood gas analyses were repeated half-hourly, the laboratory parameters 4 and $8 \mathrm{~h}$ after ICU admission. Hemodynamic monitoring was performed by continuous ECG recording, invasive blood pressure, central venous pressure measurement and monitoring of peripheral and central venous saturation. Mechanical ventilation with biphasic positive airway pressure was turned after the admission to the ICU as soon as possible to continuous positive airway ventilation with low positive end-expiratory pressure. Criteria for weaning from the ventilator included absence of bleeding signs (chest tube drainage $<100 \mathrm{ml} / \mathrm{h}$, stable haemoglobin values), stable cardiorespiratory conditions (mean arterial pressure $>60 \mathrm{mmHg}$, central venous saturation $>65$ and Horowitz index $>200$ ), and absence of high inotropic support. If patients fulfilled these criteria, sedation agents were tapered and continuous positive airway ventilation gradually decreased to a minimum level (FiO2: $35 \%$, PEEP: 5-6 mbar and ASB: 6-7 mbar). Extubation was performed in the presence of appropriate level of consciousness, adequate airway protection reflexes (cough and swallow) and in the absence of respiratory or cardiac distress. Patients were discharged from the ICU to the cardiothoracic ward at least $4 \mathrm{~h}$ after extubation but no later than 09:00 p.m. on the day of surgery. During this period, any increasing requirements for cardioactive drugs, or significant decrease in oxygen saturation ( $<90 \%$ despite oxygen mask), urine output, or level of consciousness, was considered a contraindication for discharge. In the cardiothoracic ward patients monitor surveillance has been performed for two days after the procedure via continuous ECG, peripheral oxygen saturation control and non-invasive blood pressure measurement in a room that fulfils the conditions of an intermediate care unit.

Reasons for readmission included (1) pulmonary (respiratory distress characterized by tachypnea, decrease in arterial saturation $<90 \%$, Horowitz index $<200$, use of accessory muscles or abdominal paradox, inability to clear secretion); (2) bleeding or pericardial tamponade (new onset bleeding of more than $200 \mathrm{ml} / \mathrm{h}$ or more than $800 \mathrm{ml} / 6 \mathrm{~h}$ ); (3) severe agitation requiring extended intravenous sedation; (4) upper or lower gastrointestinal bleeding requiring intervention or surgery; (5) any new permanent neurologic deficits (PNDs); (6) hemodynamic instability (any decrease in blood pressure requiring increasing use of cardioactive drugs).

\section{Data analysis}

Data are presented as frequency distributions and percentages. All continuous data are expressed as means \pm standard deviation. Categorical data are expressed as counts and proportions. Comparisons were done with 2- tailed $t$ test for means of normally distributed continuous variables and the Wilcoxon rank sum test for skewed data. Fisher exact or $\chi^{2}$ test was used to analyse differences among categorical data. Univariate and stepwise multivariate logistic regression analysis of perioperative variables for adverse outcome was performed by calculating the odds ratio (OR) with $95 \%$ confidence interval. Fourteen variables were analysed as follows: age older than 80, sex, chronic obstructive lung disease, chronic pulmonary hypertension, arterial hypertension, diabetes mellitus, cerebrovascular disease, peripheral vascular disease, chronic renal insufficiency, smoking history, ejection fraction $<30 \%$, moderate to severe mitral valve regurgitation, previous cardiac surgery and prolonged fluoroscopy time. Statistical analysis of data was conducted with commercially available software (SPSS 20.0 for Windows, SPSS Inc.).

\section{Results \\ Baseline data}

Data from all included patients were eligible for further analysis. Mean age was $79 \pm 7$ years, mean Log. EuroSCORE accounted for $24 \pm 10$, and $55 \%(n=113)$ of patients were men. Fast-track protocol could be successfully applied in 172 patients (83\%) (group S). In the remaining $17 \%(n=35)$ of TA-TAVI patients fasttrack management failed (group F). Demographic data of both groups are summarized in Table 1.

\section{Postoperative course}

Overall, 30-day mortality was $8 \%(n=17)$ and the incidence of early PND was $1 \%(n=3)$. Fast-track management failure was associated with a significant dramatic worsening of patient outcome compared to those with successful implementation of fast-track management, as reflected by an increased mortality (group S: $2 \%$ vs. group F: $40 \%, P=.001$ ) and increased postoperative complication rate such as new renal failure requiring dialysis (group S: 7 \% vs. group F: $26 \%, P=.03$ ). A detailed description of the postoperative course of both groups is presented in Table 2. Figure 1 illustrates the time to event curves for 30-day survival of both groups $\mathrm{S}$ and $\mathrm{F}$. The in-hospital stay after failed fast-track protocol was significantly longer (group S: $10 \pm 9$ days vs. group $\mathrm{F}: 19 \pm 12$ days, $P=.002$ ).

\section{Causes of fast-track failure}

Fast-track protocol failed in 35 patients (group F, 17 \%). Twenty-one patients had prolonged ICU-stay and 14 patients required readmission to the ICU within $48 \mathrm{~h}$ after initial discharge. Four out of 14 patients, died after readmission to the ICU. The remaining 10 patients could be successfully discharged to the general yard and finally from hospital in the further postoperative course. Reasons 
Table 1 Baseline demographic and clinical characteristics of group S (successful fast-track protocol) and group F (fast track protocol failure)

\begin{tabular}{|c|c|c|c|c|c|}
\hline \multirow[b]{3}{*}{ Variables } & \multicolumn{2}{|c|}{ Group S $(n=172)$} & \multicolumn{2}{|c|}{ Group F $(n=35)$} & \multirow[b]{3}{*}{$p$} \\
\hline & No. & $\%$ & No. & $\%$ & \\
\hline & & & & & \\
\hline Age (years) & $79.2 \pm 6.1$ & & $86.5 \pm 2.6$ & & 0.008 \\
\hline Male & 88 & 51 & 25 & 71 & 0.04 \\
\hline STS risk score & $8.2 \pm 6.8$ & & $11.4 \pm 8.4$ & & 0.18 \\
\hline Log. EuroSCORE & $23.2 \pm 9.7$ & & $25.8 \pm 8.3$ & & 0.46 \\
\hline Atrial fibrillation & 50 & 29 & 9 & 27 & 0.26 \\
\hline Diabetes & 34 & 20 & 12 & 35 & 0.11 \\
\hline Arterial Hypertension & 160 & 93 & 29 & 82 & 0.21 \\
\hline Pulmonary Hypertension & 115 & 67 & 26 & 75 & 0.25 \\
\hline Previous stroke & 26 & 15 & 6 & 17 & 0.15 \\
\hline Peripheral vascular disease & 46 & 27 & 14 & 40 & 0.08 \\
\hline COPD & 53 & 31 & 16 & 47 & 0.17 \\
\hline Chronic renal failure & 91 & 53 & 29 & 82 & 0.31 \\
\hline Mean ejection fraction (\%) & $47 \pm 15$ & & $31 \pm 5$ & & 0.05 \\
\hline Mean pressure gradient (echo; mmHg) & $51 \pm 19$ & & $44 \pm 27$ & & 0.14 \\
\hline Aortic valve area $(\mathrm{cm})$ & $0.66 \pm 0.22$ & & $0.72 \pm 0.48$ & & 0.09 \\
\hline Previous cardiac surgery & 50 & 29 & 12 & 35 & 0.29 \\
\hline Preop. MV regurgitation (moderate to severe) & 20 & 11 & 12 & 35 & 0.05 \\
\hline
\end{tabular}

COPD chronic obstructive pulmonary disease, Chronic renal failure $=$ glomerular filtration rate $<60 \mathrm{ml} / \mathrm{min} / 1.73 \mathrm{~m}^{2}$; EuroSCORE European system for cardiac risk evaluation, Group F fast track protocol failure, Group S successful fast-track protocol, MV mitral valve, STS risk score The Society of Thoracic Surgeons' risk sore

Table 2 Detailed postoperative course of group S (successful fast-track protocol) and group F (fast track protocol failure)

\begin{tabular}{|c|c|c|c|c|c|}
\hline \multirow[b]{3}{*}{ Variables } & \multicolumn{2}{|c|}{ Group S $(n=172)$} & \multicolumn{2}{|c|}{ Group F $(n=35)$} & \multirow[b]{3}{*}{$p$} \\
\hline & No. & $\%$ & No. & $\%$ & \\
\hline & & & & & \\
\hline Fluoroscopy time (min) & \multicolumn{2}{|l|}{$4.7 \pm 2.5$} & \multicolumn{2}{|l|}{$12.9 \pm 1.4$} & 0.04 \\
\hline Ventilation time $(h)$ & \multicolumn{2}{|l|}{$4.6 \pm 1.0$} & \multicolumn{2}{|l|}{$58 \pm 15$} & 0.04 \\
\hline \multicolumn{6}{|l|}{ Postoperative complications } \\
\hline - PM-Implantation & 9 & 5 & 0 & 0 & 0.001 \\
\hline - Early-PND & 2 & 1 & 1 & 3 & 0.13 \\
\hline - Major bleeding requiring revision & 3 & 2 & 10 & 29 & 0.02 \\
\hline - Gastrointestinal complications requiring endoscopic intervention & 1 & 1 & 5 & 14 & 0.07 \\
\hline - Acute kidney injury requiring dialysis & 12 & 7 & 9 & 26 & 0.03 \\
\hline \multicolumn{6}{|l|}{ Early outcome } \\
\hline 30-day Mortality & 3 & 2 & 14 & 40 & 0.001 \\
\hline Sepsis/MOV & 1 & 1 & 10 & 29 & \\
\hline Mesenteric ischemia & 1 & 1 & 2 & 6 & \\
\hline Arryythmia & 0 & 0 & 1 & 3 & \\
\hline Unknown & 1 & 1 & 1 & 3 & \\
\hline Hospital-stay (d) & \multicolumn{2}{|l|}{$10 \pm 9$} & \multicolumn{2}{|l|}{$19 \pm 12$} & 0.002 \\
\hline
\end{tabular}




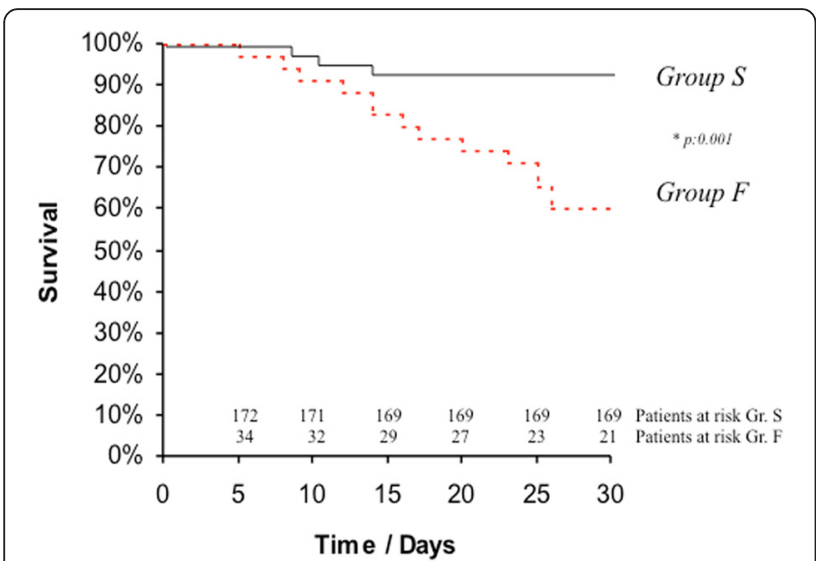

Fig. 1 Time to event curves for 30-day survival. Events were calculated with the use of Kaplan Meier methods and were compared using Log rank test. Number of patients at risk is indicated for both groups. Group $S$ denotes patients with successful fast-track protocol after TA-TAVI, whereas Group F represents those patients with fast-track management failure

for fast-track failure were as follows: respiratory failure ( $49 \%, n=17 / 35)$, major bleeding ( $29 \%, n=10 / 35)$, severe agitation (11\%, $n=4 / 35)$, gastrointestinal complications $(9 \%, n=3 / 35)$ and PND (3\%, $n=1 / 35)$. Figure 2 illustrates the causes of fast-track failure.

\section{Predictors for fast-track management failure}

Independent predictors of fast-track protocol failure were age $\geq 85$ years (OR 3.1; CI $95 \% 1.89-6.21, P=.004$ ), ejection fraction $(\mathrm{EF}) \leq 30 \%$ (OR 2.6; CI $95 \%$ 1.99-7.52,
$P=.03)$, moderate to severe preoperative mitral valve regurgitation (OR 2.7; CI $95 \%$ 1.27-6.43, $P=.008$ ) and fluoroscopy time $\geq 12 \mathrm{~min}$ (OR 2.9; CI $95 \%$ $1.28-7.46, P=.04)$.

\section{Discussion}

Prolonged sedation and ventilatory support have been practiced as an "undisputed standard" since open-heart surgery with use of $\mathrm{CPB}$ has been established in the 1950s [11, 12]. Monitoring of potential postoperative complications such as hemorrhage, myocardial ischemia and systemic inflammatory response syndrome following $\mathrm{CPB}$ was thought to be adequately performed in sedated patients under stable and easily supervised conditions [12]. Thus, anesthesiologic management with high dose opioid anesthetics made extended ventilatory support of cardiac surgical patients indispensable [13, 14]. However, cost containment and efficient resource use force the pendulum back to the debate of early extubation for patients undergoing cardiac surgery [1-3]. The improvement in anesthesia management coupled with advanced surgical techniques such as minimally invasive cardiac surgery procedures have made early extubation feasible and the concept of balanced anesthesia rather than high-dose narcotics for cardiac procedures has been introduced [4, 15-17]. Nowadays, fast track cardiac anesthesia with early extubation is widely accepted as important step in the recovery process for adult patients [3]. Due to the minimally invasive nature of TA-TAVI that eliminates sternotomy and the need for $\mathrm{CPB}$, TA-

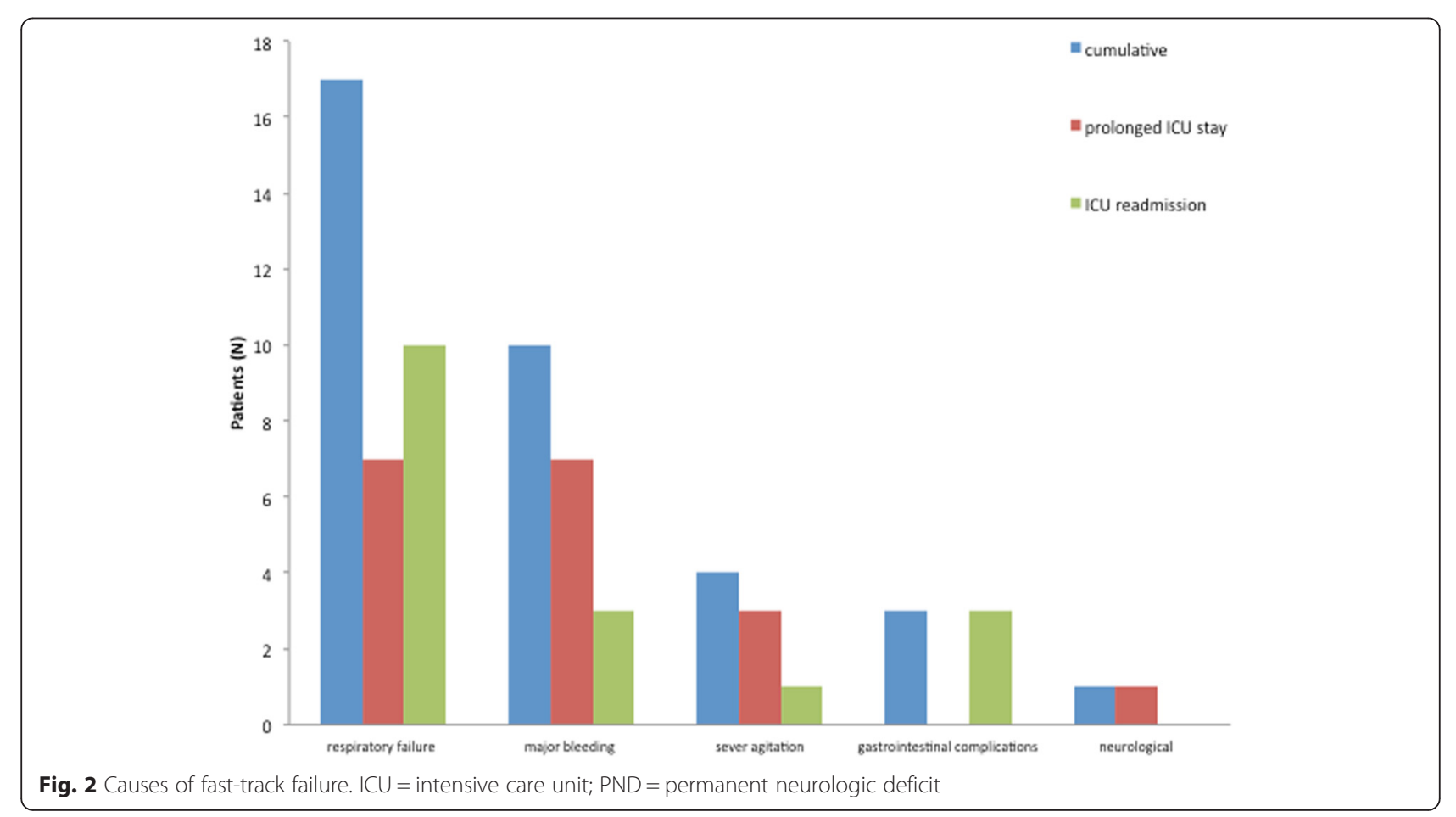


TAVI patients represent a particularly attractive cohort for fast-track management concepts [18, 19]. Thus, we sought to investigate the results after routine implementation in our institute with special focus on the risk factors that may predict fast-track protocol failure.

Undoubtedly, our TA-TAVI patients present a cohort with high perioperative risk profile, considering the mean age of $79 \pm 7$ years and the mean EuroSCORE of $24 \pm 10$ but despite their limited biological reserves fasttrack protocol could be successfully applied in $83 \%$ (Group S) of them. Since, to the best of our knowledge, no available data are published dealing specifically with fast-track management in TA-TAVI patients, only a limited comparison of our results to the literature can be facilitated. Yet, our fast-track protocol success-rate seems to be in line with data published from Haanschoten et al. in 2012, reporting a fast-track protocol success rate by 5367 low risk cardiac surgical patients of 84 \% [20]. The ultra short ICU time of Group S could not be translated in a very short hospital stay. Considering the limited biological reserves of this frail patient cohort an average of 10 days hospital stay for completion of physiotherapy treatment, enabling patients of Group $\mathrm{F}$ to be discharged either at home or at a rehabilitation center, is not a unique finding in our series. Our data regarding hospital stay are in line with the results reported from Vancouver following TA-TAVI in 178 patients with a mean length of hospital stay of 12 days [21].

The ICU readmission rates $48 \mathrm{~h}$ after the initial discharge has been also reported to be $2-5 \%$ for low risk cardiac surgery patients [22-24]. In our series, the readmission rate reached $7 \%$, which may be seen as a satisfactory result, considering the high-risk profile of our patients. Similar to other reports, the most common reason for ICU-readmission was pulmonary insufficiency [25-27]. Thus, the possibility of providing respiratory support via non-invasive ventilation in a general ward and the active follow-up of TA-TAVI patients after ICU discharge by a dedicated team of respiratory therapists has been identified as an important issue in our clinic. In this context, we believe that avoidance of volume overload in the early perioperative period as well as pain-free management and early mobilisation are of utmost importance for the optimal pulmonary recovery of fast-track TA-TAVI patients.

Another important finding of the current study is that any fast-track management failure is associated with prolonged hospital stay and dramatic worsening of patient outcome. Furthermore, failed fast-track management led to an increased postoperative complication rate such as renal failure requiring dialysis. Although speculative, a possible reason for the high mortality and morbidity in ICU readmission patients may be the early transfer of these patients to the ward. Thus it would be advantageous to identify and focus on subgroups of TATAVI patients at risk for fast-track protocol failure. Hence, despite the inherent limitations of a retrospective study, we have identified one procedural (fluoroscopy time $\geq 12 \mathrm{~min}$ ) and three preoperative clinical variables to be associated with fast-track protocol failure. Preoperative clinical variables included, age $\geq 85$ years, poor left ventricular ejection fraction (LV-EF $\leq 30 \%$ ) and moderate to severe preoperative mitral valve regurgitation. Recently, D'Onofrio and co-workers reported on the impact of preoperative mitral valve regurgitation on outcomes in 176 consecutive patients undergoing TA-TAVI in a single-centre prospective study setting. They showed that patients with a mitral valve regurgitation $\geq 2+$ had higher surgical risk and a trend towards higher in-hospital mortality [28].

While initial TA-TAVI cases in our series were performed using the first generation of the balloon expandable system significant and relentless technological advances have resulted in new and improved delivery systems and valves. On the other hand at the beginning of the TAVI era, only highest risk patients who were no suitable candidates for open surgery were considered for TAVI. With gaining experience in trancatheter approaches and increased procedural acceptance not only non-surgical-candidates but also high-risk candidates were included in our TAVI-program. Nevertheless a comparison of demographic data and outcomes of the early vs. late TA-TAVI treated patients could not reach a statistical significance in Group F in our series.

\section{Conclusions}

In conclusion, fast-track patient management following TA-TAVI is safe and successful in the majority of patients. Besides patient-related preoperative risk factors (age $\geq 85$ years, $\mathrm{EF} \leq 30 \%$ and severe preoperative mitral valve regurgitation), a technically challenging intraoperative course, as evidenced in prolonged fluoroscopy time, are independent predictors of fast-track management failure, which, in turn, is associated with prolonged hospital stay and dramatic worsening of patient outcome.

\section{Competing interests \\ Stephan Fichtlscherer is a consultant of Edwards Lifesience. The remaining authors declare that they have no competing interests.}

\section{Authors' contributions \\ NP drafted the manuscript. AEA, MT, SF, PM and CR participated in the design of the study and performed the statistical analysis. AZ and AM conceived of the study, and participated in its design and coordination and helped to draft the} manuscript. All authors read and approved the final manuscript.

\section{Author details}

'Division of Thoracic and Cardiovascular Surgery, Johann-Wolfgang-Goethe University Frankfurt/Main, Theodor Stern Kai 7, 60590 Frankfurt am Main, Germany. ${ }^{2}$ Division of Cardiology, Johann-Wolfgang-Goethe University Frankfurt/Main, Frankfurt, Germany. ${ }^{3}$ Clinic of Anesthesiology, Intensive Care 
Medicine and Pain Therapy, Goethe-University Hospital Frankfurt/Main, Frankfurt, Germany.

Received: 9 December 2015 Accepted: 4 April 2016 Published online: 11 April 2016

\section{References}

1. Barash PG, Lescovich F, Katz JD, Talner NS, Stansel Jr HC. Early extubation following pediatric cardiothoracic operation: a viable alternative. Ann Thorac Surg. 1980;29:228-33.

2. Gall Jr SA, Olsen CO, Reves JG, Mclntyre RW, Tyson Jr GS, Davis JW, et al. Beneficial effects of endotracheal extubation on ventricular performance. Implications for early extubation after cardiac operations. J Thorac Cardiovasc Surg. 1988;95:819-27.

3. Zhu F, Lee A, Chee YE. Fast-track cardiac care for adult cardiac surgical patients. Cochrane Database Syst Rev. 2012;10:CD003587. doi:10.1002/ 14651858.CD003587.

4. Smith CR, Leon MB, Mack MJ, Miller DC, Moses JW, Svensson LG, et al. Transcatheter versus surgical aortic-valve replacement in high-risk patients. N Engl J Med. 2011;364:2187-98.

5. Kodali SK, Williams MR, Smith CR. Two-year outcomes after transcatheter or surgical aortic-valve replacement. N Engl J Med. 2012;366:1686-95.

6. Dewey TM, Brown DL, Das TS, Ryan WH, Fowler JE, Hoffman SD, et al. Highrisk patients referred for transcatheter aortic valve implantation: management and outcomes. Ann Thorac Surg. 2008;86:1450-6.

7. Ben-Dor I, Gaglia Jr MA, Barbash IM, Maluenda G, Hauville C, Gonzalez MA, et al. Comparison between Society of Thoracic Surgeons score and logistic EuroSCORE for predicting mortality in patients referred for transcatheter aortic valve implantation. Cardiovasc Revasc Med. 2011;12:345-9.

8. Walther T, Simon P, Dewey T, Wimmer-Greinecker G, Falk V, Kasimir MT, et al. Transapical minimally invasive aortic valve implantation: multicenter experience. Circulation. 2007;116:240-5.

9. Papadopoulos N, llioska P, Fichtlscherer S, Lehmann R, Fernandez AB, Moritz $A$, et al. Transapical aortic valve implantation in patients with previous cardiac surgery. Ann Thorac Surg. 2014;97:37-42.

10. Doss M, Buhr EB, Martens S, Moritz A, Zierer A. Transcatheter-based aortic valve implantations at midterm: what happened to our initial patients? Ann Thorac Surg. 2012;94:1400-6.

11. Juca R, Monte L. Early tracheal extubation after coronary artery bypass grafting. J Thorac Cardiovasc Surg. 1997;1 14:687-8.

12. Sirio CA, Martich GD. Who goes to the ICU postoperatively? Chest. 1999;115: $125-9$.

13. Royse CF, Royse AG, Soeding PF. Routine immediate extubation after cardiac operation: a review of our first 100 patients. Ann Thorac Surg. 1999; 68:1326-9.

14. Silbert BS, Santamaria JD, O'Brien JL, Blyth CM, Kelly WJ, Molnar RR, et al. Early extubation following coronary artery bypass surgery: a prospective randomized controlled trial. Chest. 1998;113:1481-8.

15. Ovrum E, Tangen G, Schiott C, Dragsund S. Rapid recovery protocol applied to 5,658 consecutive "on-pump" coronary bypass patients. Ann Thorac Surg. 2000;70:2008-12.

16. Quigley RL, Reitknecht FL. A coronary artery bypass "fast-track" protocol is practical and realistic in a rural environment. Ann Thorac Surg. 1997;64:706-9.

17. Fraund S, Behnke H, Boening A, Cremer J. Immediate postoperative extubation after minimally invasive direct coronary artery surgery (MIDCAB). Interact Cardiovasc Thorac Surg. 2002;1:41-5.

18. Ducrocq G, Al-Attar N, Himbert D, Messika-Zeitoun D, lung B, Descoutures F, et al. Early and mid-term outcomes in patients undergoing transcatheter aortic valve implantation after previous coronary artery bypass grafting. Eur J Cardiothorac Surg. 2012;41:499-504.

19. Wilbring M, Tugtekin SM, Alexiou K, Simonis G, Matschke K, Kappert U. Transapical transcatheter aortic valve implantation vs conventional aortic valve replacement in high-risk patients with previous cardiac surgery: a propensity-score analysis. Eur J Cardiothorac Surg. 2013;44(1):42-7. doi:10. 1093/ejcts/ezs680.

20. Haanschoten MC, van Straten AH, ter Woorst JF, Stepaniak PS, van der Meer A, van Zundert AA, et al. Fast-track practice in cardiac surgery: results and predictors of outcome. Interact Cardiovasc Thorac Surg. 2012;15:989-94.

21. Higgins J, Ye J, Toggweiler S, Webb JG, Cheung A. Transapical aortic valve implantation: The Vancouver experience. Ann Cardiothorac Surg. 2012;1:138-44.
22. Kogan A, Cohen J, Raanani E, Sahar G, Orlov B, Singer P, et al. Readmission to the intensive care unit after "fast-track" cardiac surgery: risk factors and outcomes. Ann Thorac Surg. 2003;76:503-7.

23. Bardell T, Legare JF, Buth KJ, Hirsch GM, Ali IS. ICU readmission after cardiac surgery. Eur J Cardiothorac Surg. 2003;23:354-9.

24. Litmathe J, Kurt M, Feindt P, Gams E, Boeken U. Predictors and outcome of ICU readmission after cardiac surgery. Thorac Cardiovasc Surg. 2009;57:391-4.

25. Rosenberg AL, Watts C. Patients readmitted to ICUs* : a systematic review of risk factors and outcomes. Chest. 2000;118:492-502.

26. Chung DA, Sharples LD, Nashef SA. A case-control analysis of readmissions to the cardiac surgical intensive care unit. Eur J Cardiothorac Surg. 2002;22: 282-6.

27. Cohn WE, Sellke FW, Sirois C, Lisbon A, Johnson RG. Surgical ICU recidivism after cardiac operations. Chest. 1999;116:688-92.

28. D'Onofrio A, Gasparetto V, Napodano M, Bianco R, Tarantini G, Renier V, et al. Impact of preoperative mitral valve regurgitation on outcomes after transcatheter aortic valve implantation. Eur J Cardiothorac Surg. 2012;41: $1271-6$.

\section{Submit your next manuscript to BioMed Central and we will help you at every step:}

- We accept pre-submission inquiries

- Our selector tool helps you to find the most relevant journal

- We provide round the clock customer support

- Convenient online submission

- Thorough peer review

- Inclusion in PubMed and all major indexing services

- Maximum visibility for your research

Submit your manuscript at www.biomedcentral.com/submit
Biomed Central 